\title{
A strong shape theory with S-duality
}

\author{
by
}

Friedrich W. B a u er (Frankfurt a.M.)

\begin{abstract}
If in the classical S-category $\mathfrak{P}, 1)$ continuous mappings are replaced by compact-open strong shape (= coss) morphisms (cf. $\S 1$ or [1], §2), and 2)^-products are properly reinterpreted, then an S-duality theorem for arbitrary subsets $X \subset S^{n}$ (rather than for compact polyhedra) holds (Theorem 2.1).
\end{abstract}

0. Introduction. In a previous paper [1] we introduced the concept of coss-shape (compact-open strong shape or strong shape with compact support) and established for each $\mathbf{X}=(X, m)$, with $m \in \mathbb{Z}$ and finitedimensional $X \in$ Met (= category of separable metric spaces), i.e. for any object in an S-category $\mathfrak{P}$ (cf. $[1, \S 4]$ ), an S-dual $D \mathbf{X} \in \mathfrak{P}$ which is, up to isomorphism in $\mathfrak{P}$, uniquely determined and natural, and satisfies $D^{2} \mathbf{X} \approx \mathbf{X}$ and $\mathfrak{P}(\mathbf{X}, \mathbf{Y}) \approx \mathfrak{P}(D \mathbf{Y}, D \mathbf{X})$.

In the present paper we introduce in $\S 1$ and $\S 3$ new, additional cossmorphisms $\mathbf{X} \rightarrow \mathbf{Y} \wedge \mathbf{Z}$ and $\mathbf{X} \pi \mathbf{Y} \rightarrow \mathbf{Z}$ ( $\mathbf{Y} \pi \mathbf{Z}$ and $\mathbf{X} \pi \mathbf{Y}$ are pairs of objects in $\mathfrak{P}$ ) which for special choices of $\mathbf{X}, \mathbf{Y}, \mathbf{Z}$ can be interpreted as coss-morphisms between $\mathbf{X}$ and $\mathbf{Y} \wedge \mathbf{Z} \in \mathfrak{P}$ (cf. §3) resp. $\mathbf{X} \wedge \mathbf{Y}$ and $\mathbf{Z}$, or sometimes even as strong shape morphisms (1.2 and $\S 5)$.

The main objective of this paper is the verification of Theorem 2.1 asserting the existence of a natural isomorphism

$$
\{\mathbf{X} \pi \mathbf{Y}, \mathbf{Z}\}_{c} \approx\{\mathbf{X}, D \mathbf{Y} \pi \mathbf{Z}\}_{c}
$$

$\left(\{\ldots, \ldots\}_{c}\right.$ denoting the respective sets of coss-morphisms in the S-category, cf. $\S 3)$.

Among the corollaries in $\S 2$ we recover the main theorem of [1] and two other S-duality theorems for special choices of $\mathbf{X}, \mathbf{Y}, \mathbf{Z}$. In particular, classical S-duality (cf. [9], [10]) turns out to be a corollary of (1) (cf. $\S 2(8)$ ).

1991 Mathematics Subject Classification: Primary 55P25, 55P55; Secondary 55N20, $55 \mathrm{M} 05$.

Key words and phrases: S-duality, Alexander duality, compact-open strong shape, virtual spaces. 
All these corollaries have in common that the concept of new cossmorphisms as introduced in $\S 1$ does not enter, but that we get along with either coss-morphisms between spaces (2.2), strong shape morphisms or even continuous mappings (2.3).

In $\S 3$ we treat the S-duality operator $D$ and introduce stable cossmorphisms, while in $\S 4$ we provide the necessary Alexander duality theorems from which all kinds of S-duality are derived.

The reader is supposed to be familiar with [1]: Not only do we retain all notations from [1] but if a construction or a proof has a counterpart in [1], then we do not resume the arguments, referring instead to the corresponding statement or assertion there.

1. The coss-morphisms $\alpha: X \bar{\wedge} Y \rightarrow Z, \beta: X \rightarrow Y \bar{\wedge} Z$. Before we present the definition of two kinds of new coss-morphisms, we have to set up the categories of topological spaces in which we are going to operate. This is primarily done by referring to $[1, \S 1]$, with the only exception that, in contrast to [1], we are working with based spaces. Thus we understand by Met the category of based separable metrizable spaces, with based continuous mappings. Very rarely we encounter unbased spaces (specifically, in the formulation of Alexander duality theorems in $\S 4$ ). Then $X \in$ Met always means, by an abuse of notation, $X^{+} \in$ Met.

By an ANR (or a good space) we understand an ANR in Met. The same pertains to compact spaces. Hence by a space we always mean a space in Met.

By a classical theorem, all spaces allow an embedding in the Hilbert cube, resp. in some $S^{n}$ whenever they are finite-dimensional.

In dealing with smash products $X \wedge Y$ between spaces in Met, we face the same kind of problem which we settled in $[1, \S 1]$ concerning cones and suspensions:

Since $X \wedge Y$ with the customary topology is not necessarily an object in Met unless $X$ and $Y$ are compact, we have to adjust the topology. This can be done, for example, in the finite-dimensional case, by taking embeddings $X \subset S^{n}, Y \subset S^{m}$ and equipping $X \wedge Y$ with the subset topology of $S^{n} \wedge S^{m}=S^{n+m}$; in general we employ any compacta $K, L$ satisfying $X \subset K, Y \subset L$. This topology turns out to be independent of the specific embedding, which can be easily realized by displaying an intrinsic definition of this topology in the same way as we did in $[1, \S 1]$.

In particular, both concepts of $\wedge$-products are always homotopy equivalent (details are as in $[1, \S 1]$ ). We always mean this topology whenever we write $X \wedge Y$.

Let $P, Q$ be ANRs. Then $P \wedge Q$ is again an ANR. The quickest way to confirm that is an inspection of the proof of $[5$, Theorem 8.2, p. 406] 
by $\mathrm{O}$. Hanner, dealing with the question whether a quotient space $P / Q$ of an ANR $P$, with $Q$ a closed subspace and an ANR, is again an ANR. The answer is in the affirmative under some compactness assumption, which is only needed in order to guarantee that the resulting space is in Met, which in our case is ensured by our convention concerning the topology of $P \wedge Q$.

Thus we have $P \wedge Q \subset S^{n} \wedge S^{m}=S^{n+m}$, which is an ANR whenever $P$ and $Q$ are finite-dimensional ANRs.

In $[1, \S 2]$ we introduced the concept of coss-morphisms between spaces. This carries over immediately to based spaces, simply by employing based continuous and based strong shape morphisms in the constructions.

In addition to strong shape morphisms between compact spaces $\bar{f}: X \rightarrow$ $Y$, we need strong shape mappings of the form $X \rightarrow Y \bar{\wedge} R$ and $X \rightarrow R \bar{\wedge} Y$, where $Y$ is compact, $R$ good and $X$ either compact or an ANR. This kind of strong shape morphisms is already known from [3] and recorded in $\S 4$.

Let $X, Y, Z$ be spaces and $X \bar{\wedge} Y, Y \bar{\wedge} Z$ pairs of spaces. Then we introduce new morphisms $\alpha: X \bar{\wedge} Y \rightarrow Z$ and $\beta: X \rightarrow Y \wedge Z$ in the following way:

Let $P, Q, R, \widetilde{P}, \widetilde{Q}$ be good spaces (i.e. ANRs in Met), $X^{\prime}, Y^{\prime}, Z^{\prime}, \widetilde{Y}$ compact in Met, and $r: X \rightarrow P, s: Y \rightarrow Q, t: Z \rightarrow R, a: X^{\prime} \rightarrow X$, $b: Y^{\prime} \rightarrow Y, c: Z^{\prime} \rightarrow Z, \widetilde{s}: \widetilde{Y} \rightarrow Q, \widetilde{a}: X^{\prime} \rightarrow \widetilde{P}$ and $\widetilde{b}: Y^{\prime} \rightarrow \widetilde{Q}$ continuous mappings.

We consider diagrams $\sigma=\sigma\left(a, b, c, r, s, t, \widetilde{a}, \widetilde{b}, g_{1}, g_{2}, \bar{f}\right)$

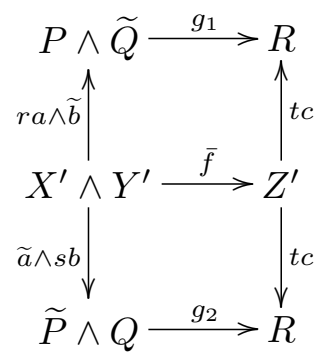

with $g_{1}, g_{2}$ being stable homotopy classes of continuous morphisms and $\bar{f}$ a stable homotopy class of a strong shape morphism (cf. [1, $\S 2]$, the associated definition of a coss-morphism between spaces), which are stably homotopy commutative. This means that $\bar{f}\left(\Sigma^{k}(t c)\right)$ (i.e. the strong shape morphism $\bar{f}$ evaluated at $\left.\Sigma^{k}(t c)\right)$ and $g_{1}\left(\Sigma^{k}(r a) \wedge \widetilde{b}\right)\left(\right.$ resp. $\left.g_{2}\left(\Sigma^{k} \widetilde{a} \wedge s b\right)\right)$ are stably homotopic. In other words, there exist natural numbers $l, m$ such that

$\Sigma^{l} \bar{f}\left(\Sigma^{k}(t c)\right) \simeq \Sigma^{l} g_{2}\left(\Sigma^{l+k}(\widetilde{a} \wedge s b)\right), \quad \Sigma^{m} \bar{f}\left(\Sigma^{k}(t c)\right) \simeq \Sigma^{m} g_{1}\left(\Sigma^{k+m}(r a) \wedge \widetilde{b}\right)$.

Thus, by taking appropriate representatives $\bar{f}, g_{1}, g_{2}$, we can assume (as in $[1, \S 2])$ that for suitable $k$,

$$
\bar{f}\left(\Sigma^{k}(t c)\right) \simeq g_{2}\left(\Sigma^{k}(\widetilde{a} \wedge s b)\right), \quad \bar{f}\left(\Sigma^{k}(t c)\right) \simeq g_{1}\left(\Sigma^{k}(r a) \wedge \widetilde{b}\right) .
$$


Let $T(a, b, t)$ be the class of all such diagrams $\left(a, b, t\right.$ fixed). Then $\sigma_{1}, \sigma_{2} \in$ $T(a, b, t)$ are equivalent whenever we detect continuous mappings $P_{1} \rightarrow P_{2}$, $Z_{1}^{\prime} \rightarrow Z_{2}^{\prime}, \widetilde{Q}_{1} \rightarrow \widetilde{Q}_{2}, \widetilde{P}_{1} \rightarrow \widetilde{P}_{2}$ and $Q_{1} \rightarrow Q_{2}$ rendering all diagrams which result from inserting these mappings into $\sigma_{1}, \sigma_{2}$ stably commutative.

This generates (as in $[1, \S 2]$ ) an equivalence relation. The set of equivalence classes is denoted by

$$
T[a, b, c]=\lim T(a, b, t) .
$$

Now we proceed as in $[1, \S 2]$, considering assignments $\alpha$ which assign to $a, b, t$ an element in $T[a, b, t]$ such that for a commutative diagram

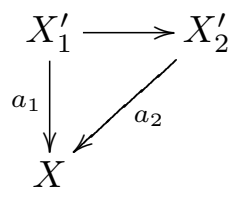

the diagram resulting from $\alpha\left(a_{2}, b, t\right)$ by inserting (2) (in an obvious way) is equal to $\alpha\left(a_{1}, b, t\right)$ (in $T\left[a_{1}, b, t\right]$ ).

The same is required for commutative diagrams

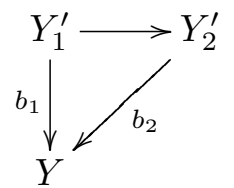

and

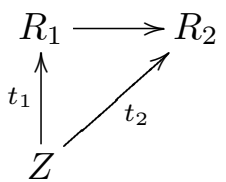

This procedure is some kind of inverse limit and, in complete analogy to [1, Definition 2.1], we set up

Definition 1.1. The set of these $\alpha(X, Y, Z$ fixed in Met) is denoted by

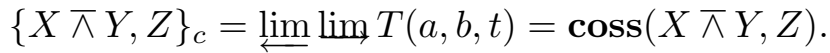

In the same way we define mappings $\beta: X \rightarrow Y \wedge Z$ : We consider stably commutative diagrams

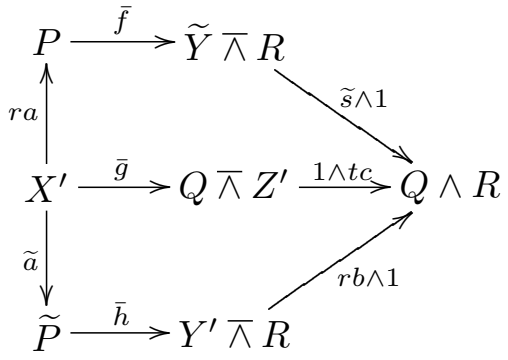

and denote by $T(a, t, s)$ all those diagrams with fixed $a, t, s$. Then we form again the equivalence classes

$$
T[a, t, s]=\lim T(a, t, s)
$$


and declare a morphism $\beta: X \rightarrow Y \nwarrow Z$ to be an assignment which assigns to each $(a, t, s)$ an element $\beta(a, t, s) \in T[a, t, s]$ satisfying obvious naturality properties, providing us finally with

DEFINITION $1.1^{\star}$.

$$
\{X, Y \wedge Z\}_{c}=\lim _{\longleftarrow} \lim (a, t, s)=\operatorname{coss}(X, Y \pi Z) .
$$

We do not define all kinds of compositions between new or old cossmorphisms but confine ourselves to the following cases:

1) Let $\beta: X \rightarrow Y \pi Z, \gamma \in \operatorname{coss}\left(Y, Y_{1}\right)$ and $\eta \in \operatorname{coss}\left(Z, Z_{1}\right)$. Then the composition $(\gamma \bar{\wedge} \eta) \beta \in \operatorname{coss}\left(X, Y_{1} \bar{\wedge} Z_{1}\right)$ is established in the same way as the composition of coss-morphisms between spaces in $[1, \S 2]$.

The same pertains to the compositions:

2) $X_{1} \pi Y_{1} \stackrel{\gamma \bar{\wedge} \eta}{\longrightarrow} X \pi Y \stackrel{\alpha}{\rightarrow} Z$,

3) $X_{1} \stackrel{\gamma}{\rightarrow} X \stackrel{\beta}{\rightarrow} Y \pi Z$,

4) $X \pi Y \stackrel{\alpha}{\rightarrow} Z \stackrel{\eta}{\rightarrow} Z_{1}$.

We do not define morphisms

$$
X \bar{\wedge} Y \stackrel{\alpha}{\rightarrow} Z \stackrel{\beta}{\rightarrow} X_{1} \bar{\wedge} Y_{1} \quad \text { nor } \quad X \stackrel{\beta}{\rightarrow} Y \bar{\wedge} Z \stackrel{\alpha}{\rightarrow} X_{1} .
$$

So coss-morphisms only form a category for spaces (that was already accomplished in [1]), for pairs of spaces, but not for spaces and pairs of spaces together (cf. remark at the end of this section).

Proposition 1.2. If $Y$ is a compact $A N R$ in Met, then for all $X, Z \in$ Met, we have natural equivalences

$$
\begin{aligned}
& \operatorname{coss}(X \wedge Y, Z) \approx \operatorname{coss}(X \wedge Y, Z), \\
& \operatorname{coss}(X, Y \wedge Z) \approx \operatorname{coss}(X, Y \wedge Z) .
\end{aligned}
$$

Here on the left hand sides we have new coss-morphisms (between spaces), and on the right hand sides the old ones.

Proof. 1) Denote by $A \widetilde{\wedge} B$ the $\wedge$-product between two spaces in Met with the ordinary topology (i.e. $A \widetilde{\wedge} B=A \times B / A \vee B$ ). Then we have:

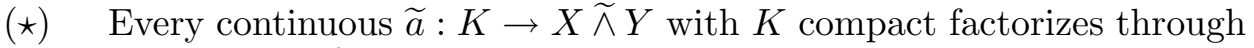
$X^{\prime} \wedge Y=X^{\prime} \wedge Y$ with $X^{\prime} \subset X$ compact.

This has (for $B=Y=S^{1}$ ) already been verified and used in the proof of Lemma 2.7 in [1]. The proof of $(\star)$ follows the same line.

2) A morphism $\alpha: X \wedge Y \rightarrow Z$ determines mappings

$$
P \wedge \widetilde{Q} \rightarrow R, \quad \widetilde{P} \wedge Q \rightarrow R, \quad \bar{f}: X^{\prime} \wedge Y^{\prime} \rightarrow Z^{\prime},
$$


hence, we are allowed to set $Y=Y^{\prime}=Q=\widetilde{Q}=\widetilde{Y}$; a mapping $g: P \wedge Q \rightarrow$ $R$, and therefore the diagram (1) reduces to

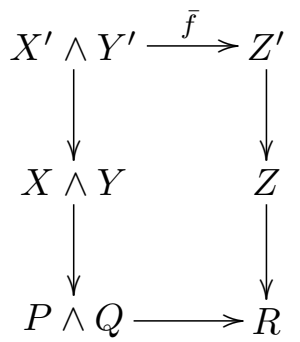

Suppose $a: K \rightarrow X \wedge Y$ is given, with $K$ compact. Then according to $(\star)$ we have a homotopy commutative diagram

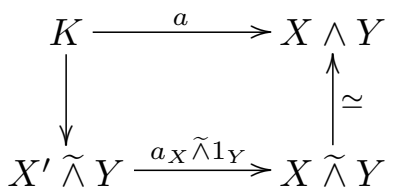

employing the homotopy equivalence between $X \widetilde{\wedge} Y$ and $X \wedge Y$ (the $\wedge$ product with the ordinary and that with the new topology). As a result we find

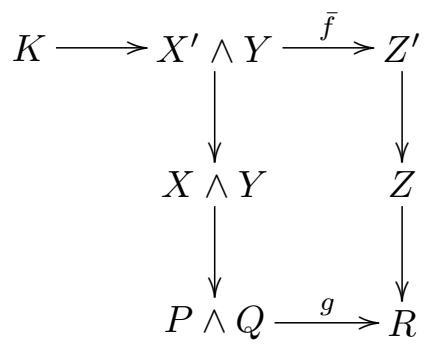

where $\bar{f}, g$ stem from (6). In view of Definition 2.1 of [1] this gives rise to a coss-morphism $\widehat{\alpha}: X \wedge Y \rightarrow Z$, hence to a transformation

$$
\operatorname{coss}(X \wedge Y, Z) \rightarrow \operatorname{coss}(X \wedge Y, Z) .
$$

3) Suppose we start with a coss-morphism $\beta: X \wedge Y \rightarrow Z$, and let $a: X^{\prime} \rightarrow X$ with $X^{\prime}$ compact and $r: Z \rightarrow R$. Then we have a stably commutative diagram

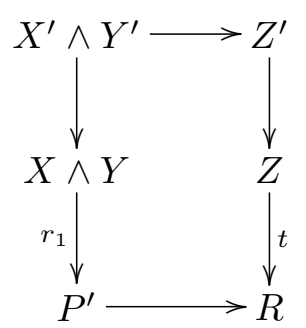


We have $\widetilde{r}_{1}: X \widetilde{\wedge} Y \rightarrow P^{\prime}, P^{\prime} \in \mathbf{A N R}$, satisfying

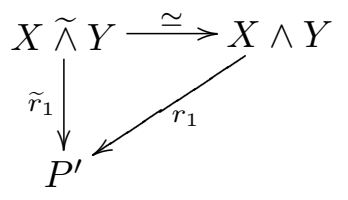

and a homotopy commutative diagram

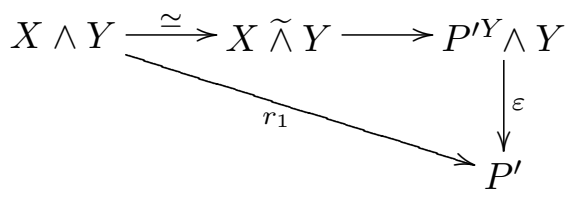

where $\varepsilon$ is the evaluation map.

So we finally get a stably commutative diagram

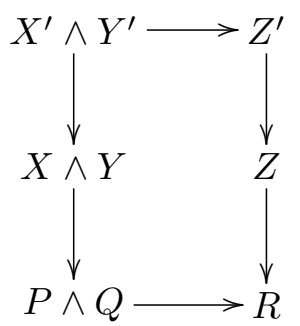

for suitable $P$ with $Q=Y$. Although we cannot simply implement $P=P^{\prime Y}$ right away (because this is not necessarily an object of Met), there exists a $P$, e.g. an open neighborhood of $X$ in a Hilbert cube containing $X$, over which $X \rightarrow P^{\prime Y}$ extends. This provides us with the mapping (5) giving rise to a $\check{\beta} \in \operatorname{coss}(X \pi Y, Z)$ such that $\hat{\tilde{\beta}}=\beta$ and $\check{\hat{\alpha}}=\alpha$. The naturality is immediate. The verification of (4) is similar.

Proposition 1.3. For all $X, Y, Z$ in Met we have a natural equivalence

$$
\operatorname{coss}(X \pi Y, Z) \approx \operatorname{coss}(Y \AA X, Z) \text {. }
$$

Proof. A morphism $\alpha: X \bar{\wedge} Y \rightarrow Z$ is defined by means of strong shape resp. continuous mappings $X^{\prime} \wedge Y^{\prime} \rightarrow Z^{\prime}, \widetilde{P} \wedge Q \rightarrow R, P \wedge \widetilde{Q} \rightarrow R$, exhibiting a symmetry in $X$ and $Y$.

R e $\mathrm{m}$ ar $\mathrm{k}$. There is no analogous equivalence between $\operatorname{coss}(X, Y \bar{\wedge} Z)$ and $\operatorname{coss}(X, Z \pi Y)$, because we are considering in Definition $1.1^{\star}$, in particular in diagram $\left(1^{\star}\right)$, all strong shape morphisms $X^{\prime} \rightarrow Q \wedge Z$ but only those strong shape morphisms $X^{\prime} \rightarrow Y^{\prime} \wedge R$ which allow an extension over a good $\widetilde{P}$. Moreover, $\bar{f}: P \rightarrow \widetilde{Y} \wedge R$ has no counterpart in this definition for $Y, Z$ interchanged. 
In analogy to definition $2.1,2)$ of [1] we are able to fix embeddings $X \subset P, Y \subset Q, Z \subset R$ (in practice $P, Q, R$ will be certain $n$-spheres) and deal with diagrams (1) resp. $\left(1^{\star}\right)$ where all $a: X^{\prime} \subset X, b: Y^{\prime} \subset Y$, $c: Z^{\prime} \subset Z, r: X \subset U, s: Y \subset V, t: Z \subset W, \widetilde{a}: X^{\prime} \subset \widetilde{U}, \widetilde{b}: Y^{\prime} \subset \widetilde{V}$ and $\widetilde{s}: \widetilde{Y} \subset V$ are inclusions and $U, \widetilde{U} \subset P, V, \widetilde{V} \subset Q$ and $W \subset R$ are open subsets. The set of all such squares is denoted by $T^{\prime}$. In this case, for a diagram $\sigma$ of the form (1) or $\left(1^{\star}\right)$ we prefer to write $\sigma\left(X^{\prime}, Y^{\prime}, \ldots\right)$ instead of $\sigma(a, b, \ldots)$.

Proposition 1.4. There is an equivalence between $\operatorname{coss}(X, Y \bar{\wedge} Z)$ and $\underset{\lim }{\longleftarrow} T^{\prime}\left(X^{\prime}, V, W\right)$, and between $\operatorname{coss}(X \bar{\wedge} Y, Z)$ and $\lim _{\varlimsup} \lim T^{\prime}\left(X^{\prime}, Y^{\prime}, W\right)$.

The proof follows exactly the same pattern as that of Lemma 2.6 in [1] which can be immediately translated to the present case.

We would like to have, analogous to Lemma 2.7 of [1], a stability proposition for the new coss-morphisms.

To this end, for a based space $X=\left(X, x_{0}\right) \in$ Met we define the suspension $\Sigma X=(\Sigma X, \star)$ as the unreduced suspension of $X$ with top vertex $\star$ of $\Sigma X$ as base point and observe:

Lemma 1.5. For good $X$ in $\mathbf{M e t}_{h}$, resp. compact $X$ in $\overline{\mathbf{K}}_{h}=\overline{\mathbf{C o m}}_{h}$, we have a homotopy equivalence between $(\Sigma X, \star)$ and $\left(\Sigma X, x_{0}\right)=\widehat{\Sigma} X$ in $\mathbf{M e t}_{h}$, resp. in $\overline{\mathbf{K}}_{h}$.

Proof. The spaces $\left(X, x_{0}\right)$ are well-pointed in both categories (cf. [3, A10, p. 301]) so that the contention for $\widehat{\Sigma}$ and $\widetilde{\Sigma}$ follows by standard arguments. However, $\widetilde{\Sigma}$ and $\Sigma$ (resp. $\widehat{\widetilde{\Sigma}}$ and $\widehat{\Sigma}$ ) are known to be homotopy equivalent (cf. $[1, \S 1])$.

So we obtain:

LEMMA 1.6. There are equivalences between $\operatorname{coss}(\Sigma X \pi Y, \Sigma Z)$ and $\operatorname{coss}(X \pi \Sigma Y, \Sigma Z)($ resp. $\operatorname{coss}(\Sigma X, \Sigma Y \wedge Z)$ and $\operatorname{coss}(\Sigma X, Y \wedge \Sigma Z))$ and the related morphism sets, where all or some $\Sigma$ are replaced by $\widehat{\Sigma}$.

Proof. Follows (using the same argument as in the proof of [1, Lemma 2.7]) from the definition of these morphisms (involving only good or compact spaces) because these equivalences exist for strong shape resp. continuous mappings and because of 1.5.

Proposition 1.7. We have natural equivalences

$$
\Sigma_{*}: \operatorname{coss}(\Sigma X, \Sigma Y \pi Z) \approx \operatorname{coss}(\Sigma X, Y \wedge \Sigma Z) \approx \operatorname{coss}(X, Y \pi Z)
$$

and

$$
\Sigma_{*}: \operatorname{coss}(\Sigma X \pi Y, \Sigma Z) \approx \operatorname{coss}(X \pi \Sigma Y, \Sigma Z) \approx \operatorname{coss}(X \pi Y, Z) .
$$

The same holds for $\widehat{\Sigma}$ replacing $\Sigma$. 
Pr o of. These equivalences are established by the same arguments leading to Lemma 2.7 of [1].

Proposition 1.8. $\operatorname{coss}(X \pi Y, Z)$ and $\operatorname{coss}(X, Y \pi Z)$ carry a natural abelian group structure, turning both equivalences $\Sigma_{*}$ from 1.7 as well as those in 1.2 and 1.3 into isomorphisms of abelian groups.

Proof. Follows in the same way as 2.8 of [1].

Remark. We could easily repair the fact that coss with these new morphisms $X \rightarrow Y \wedge Z$ and $X \pi Y \rightarrow Z$ is not any more a category: We distinguish between two different types of pairs, say $X \pi Y$ (for outgoing

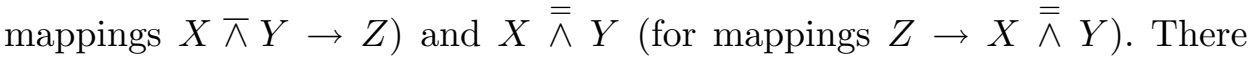
are no mappings starting from $X \overline{\bar{\wedge}} Y$ with target being a space, resp. no mappings going out from a space into $X \pi Y$ and no mappings between $X \bar{\wedge} Y$ and $\bar{\wedge}$-products and vice versa.

Such pairs are customarily called virtual spaces to distinguish them from real spaces (i.e. spaces in Met).

2. The S-duality isomorphism. We now formulate and prove the main theorem of this paper. Concerning the definition of $D$, the S-category $\mathfrak{P}$ and the abelian groups $\{\mathbf{X} \wedge \mathbf{Y}, \mathbf{Z}\}_{c},\{\mathbf{X}, \mathbf{Y} \wedge \mathbf{Z}\}_{c}$, we refer to $\S 3$.

Theorem 2.1. For $\mathbf{X}, \mathbf{Y}, \mathbf{Z} \in \mathfrak{P}$ there exists an isomorphism of abelian groups

$$
\phi:\{\mathbf{X} \pi \mathbf{Y}, \mathbf{Z}\}_{c} \approx\{\mathbf{X}, D \mathbf{Y} \wedge \mathbf{Z}\}_{c}
$$

which is natural with respect to morphisms in $\mathfrak{P}$.

Proof. Recall that a morphism $\alpha \in\{\mathbf{X} \wedge \mathbf{Y}, \mathbf{Z}\}_{c}$ is determined by diagrams $\S 1(1)$, hence by mappings $\bar{f}: X^{\prime} \wedge Y^{\prime} \rightarrow Z^{\prime}, g_{1}: P \wedge \widetilde{Q} \rightarrow R$ and $g_{2}: \widetilde{P} \wedge Q \rightarrow R$. Moreover, in view of 1.4 we can assume without loss of generality that $X \subset S^{n}, Y \subset S^{m}$ and $Z \subset S^{k}$ are given embeddings and that 1) $X^{\prime} \subset X, Y^{\prime} \subset Y, Z^{\prime} \subset Z, X^{\prime} \subset \widetilde{P}$ and $Y^{\prime} \subset \widetilde{Q}$ are all inclusions, and 2) $P=U, Q=V, R=W, \widetilde{P}=\widetilde{U}$ and $\widetilde{Q}=\widetilde{V}$ are open subsets of the given spheres. In the same way we realize that $\boldsymbol{\beta} \in\{\mathbf{X}, \mathbf{Y} \wedge \mathbf{Z}\}_{c}$ is associated with mappings $\bar{g}: X \rightarrow V \bar{\wedge} Z^{\prime}, \bar{h}: \widetilde{P} \rightarrow Y^{\prime} \wedge W$ and $\bar{f}^{\prime}: P \rightarrow \widetilde{Y} \wedge W$. The equivalence $\phi$ is established by detecting natural bijections between $\left(\bar{f}, g_{1}, g_{2}\right)$ and $\left(\bar{g}, \bar{f}^{\prime}, \bar{h}\right)$. To this end, using the terminology of $\S 4$, we employ the Alexander duality isomorphisms

$$
\begin{aligned}
& \overline{\left\{X^{\prime} \wedge Y^{\prime}, Z^{\prime}\right\}} \stackrel{\text { II }}{\left.\approx S^{0},\left(D X^{\prime} \wedge D Y^{\prime}\right) \bar{\wedge} Z^{\prime}\right\}} \\
& \approx \overline{\left\{S^{0}, D X^{\prime} \bar{\wedge}\left(D Y^{\prime} \bar{\wedge} Z^{\prime}\right)\right\}} \stackrel{\operatorname{III}}{\approx\left\{X^{\prime}, D Y^{\prime} \bar{\wedge} Z^{\prime}\right\}}, \\
& \{\widetilde{U} \wedge V, W\} \stackrel{\mathrm{I}}{\approx} \overline{\left\{S^{0},(D \widetilde{U} \wedge D V) \wedge W\right\}} \stackrel{\mathrm{I}}{\approx} \overline{\{\widetilde{U}, D V \bar{\wedge} W\}},
\end{aligned}
$$




$$
\{U \wedge V, W\} \stackrel{\mathrm{I}}{\left.\approx S^{0},(D U \wedge D \widetilde{V}) \pi W\right\}} \stackrel{\mathrm{I}}{\approx} \overline{\{U, D \widetilde{V} \pi W\}}
$$

The numbers over the $\approx$ signs refer to the specific associated Alexander duality isomorphisms of $\S 4$ which are applied. Furthermore, we have made use of Proposition 3.8; moreover, 5.2 ensures that

$$
\left\{X^{\prime} \pi Y^{\prime}, Z^{\prime}\right\}_{c} \approx \overline{\left\{X^{\prime} \wedge Y^{\prime}, Z^{\prime}\right\}}
$$

We observe that $D Y^{\prime}$ is a $V, D \widetilde{Q}$ a $\widetilde{Y}$ and $D V$ a $V^{\prime}$ for $D Y$ (instead of $Y$ ).

Let $\boldsymbol{\alpha} \in\{\mathbf{X} \backslash \mathbf{Y}, \mathbf{Z}\}_{c}$, i.e. $\alpha \in \operatorname{coss}\left(\Sigma^{k} X \bar{\wedge} Y, \Sigma^{l} Z\right)$ for suitable $k, l$. Then $\alpha\left(X^{\prime}, Y^{\prime}, W\right)$ determines a triple $\bar{f}, g_{1}, g_{2}$ which, in turn, by means of (2), (3), (4), gives rise to a triple $\bar{g}, \bar{f}^{\prime}, \bar{h}$, hence, according to the naturality properties of the Alexander duality isomorphisms I-III (cf. 4.5), to a $\beta\left(X^{\prime}, D Y^{\prime}, W\right)$.

This establishes a transformation $\phi$ which can be obviously inverted, yielding a $\phi^{-1}$. The naturality of $\phi$ follows easily from the naturality properties of the Alexander duality isomorphisms involved (cf. 4.5). The additivity of $\phi$ is also immediate (cf. 3.5 concerning the abelian group structure of $\left.\{\ldots, \ldots\}_{c}\right)$.

Corollary 2.2. For $\mathbf{X}, \mathbf{Y} \in \mathfrak{P}$ we have natural isomorphims

$$
\{\mathbf{X}, \mathbf{Y}\}_{c} \approx\{D \mathbf{Y}, D \mathbf{X}\}_{c} .
$$

Proof. Recall that $\{\mathbf{X} \pi \mathbf{Y}, \mathbf{Z}\}_{c}$ is symmetrical in $\mathbf{X}$ and $\mathbf{Y}$ (cf. 2.3 for spaces, which implies the contention for objects in $\mathfrak{P})$. So we calculate:

$$
\{\mathbf{X}, \mathbf{Y}\}_{c} \approx\left\{\mathbf{X} \wedge D \mathbf{Y}, \mathbf{S}^{0}\right\}_{c} \approx\left\{D \mathbf{Y} \wedge \mathbf{X}, \mathbf{S}^{0}\right\}_{c} \approx\{D \mathbf{Y}, D \mathbf{X}\}_{c}
$$

$\left(\mathbf{S}^{0}=\Sigma^{-n}\left(S^{n}, 0\right)\right.$ for suitable $\left.n\right)$. The first and the third isomorphisms stem from Theorem 2.1 .

Remarks. 1) Corollary 2.2 for unbased spaces is the main subject of [1] (Theorem 4.3). It could be easily achieved (without involving 2.1) by adapting the original proof to the case of based spaces.

2) We could extend the definition of $D$ to the case of pairs of spaces by setting

$$
D(X \pi Y)=D X \pi D Y, \quad D(\mathbf{X} \pi \mathbf{Y})=D \mathbf{X} \pi D \mathbf{Y},
$$

furnishing us with an isomorphism

$$
D^{2}(\mathbf{X} \pi \mathbf{Y})=D(D \mathbf{X} \pi D \mathbf{Y}) \approx \mathbf{X} \pi \mathbf{Y} .
$$

However, we have in general

$$
\begin{gathered}
\{D(\mathbf{X} \wedge \mathbf{Y}), D \mathbf{Z}\}_{c} \not \approx\{\mathbf{Z}, \mathbf{X} \pi \mathbf{Y}\}_{c}, \\
\left\{D(\mathbf{X} \pi \mathbf{Y}), D\left(\mathbf{X}_{1} \wedge \mathbf{Y}_{1}\right\}_{c} \not \approx\left\{\mathbf{X}_{1} \pi \mathbf{Y}_{1}, \mathbf{X} \wedge \mathbf{Y}\right\}_{c},\right.
\end{gathered}
$$

because $\{\mathbf{X}, \mathbf{Y} \wedge \mathbf{Z}\}_{c}$ is not symmetrical in $\mathbf{Y}$ and $\mathbf{Z}$, unless we impose restrictions on the objects involved.

Let $\mathfrak{P}^{\prime}$ and $\overline{\mathbf{K}}_{\mathfrak{P}}$ be defined as in $\S 3$ with $\mathbf{X} \wedge \mathbf{Y}$ as in $\S 3(7)$. 
Corollary 2.3. 1) If $\mathbf{X}, \mathbf{Y}, \mathbf{Z}$ are compact, then we have a natural equivalence

$$
\overline{\mathbf{K}}_{\mathfrak{P}}(\mathbf{X} \wedge \mathbf{Y}, \mathbf{Z}) \approx \overline{\mathbf{K}}_{\mathfrak{P}}(\mathbf{X}, D \mathbf{Y} \wedge \mathbf{Z}) .
$$

2) If $\mathbf{X}, \mathbf{Y}, \mathbf{Z}$ are good and $\mathbf{X}$ or $\mathbf{Y}$ also compact, then we have a natural equivalence

$$
\mathfrak{P}^{\prime}(\mathbf{X} \wedge \mathbf{Y}, \mathbf{Z}) \approx \overline{\mathbf{K}}_{\mathfrak{P}}(\mathbf{X}, D \mathbf{Y} \wedge \mathbf{Z})
$$

Pr o of. Follows immediately from the stabilized versions of 5.1 and 5.2.

Remarks. 1) The equivalences (6) and (7) can both be regarded as extensions of Theorem 1.1 of [2] in the same sense as Theorem 2.1 is an extension of Corollary 2.2 and Theorem 4.3 of [1]. The abelian groups involved do not require coss-morphisms but merely strong shape or continuous mappings. Moreover, on the left hand side we find ordinary $\wedge$-products.

2) Assuming that $\mathbf{X}, \mathbf{Y}, \mathbf{Z}$ are good but $\mathbf{Y}$ also compact, we obtain again a good and compact $D \mathbf{Y}$ (up to isomorphism), hence

$$
\overline{\mathbf{K}}_{\mathfrak{P}}(\mathbf{X}, D \mathbf{Y} \pi \mathbf{Z}) \approx \mathfrak{P}^{\prime}(\mathbf{X}, D \mathbf{Y} \pi \mathbf{Z}) .
$$

As a result, (7) yields

$$
\mathfrak{P}^{\prime}(\mathbf{X} \wedge \mathbf{Y}, \mathbf{Z}) \approx \mathfrak{P}^{\prime}(\mathbf{X}, D \mathbf{Y} \wedge \mathbf{Z}),
$$

which is ordinary, classical S-duality (cf. [8, p. 18, Theorem 19] and [9]).

3) Suppose that $\mathbf{X}$ and $\mathbf{Z}$ are compact, $\mathbf{Y}=\mathbf{S}^{0}$ (i.e. compact and good). Then we calculate

$$
\begin{aligned}
\mathfrak{P}^{\prime}(D \mathbf{X}, D \mathbf{Z}) & \approx \mathfrak{P}^{\prime}\left(S^{0} \wedge D \mathbf{X}, D \mathbf{Z}\right) \stackrel{(7)}{\approx} \overline{\mathbf{K}}_{\mathfrak{P}}\left(S^{0}, \mathbf{X} \pi D \mathbf{Z}\right) \\
& \approx \overline{\mathbf{K}}_{\mathfrak{P}}\left(S^{0}, D \mathbf{Z} \bar{\wedge} \mathbf{X}\right) \stackrel{(6)}{\approx} \overline{\mathbf{K}}_{\mathfrak{P}}\left(S^{0} \wedge \mathbf{Z}, \mathbf{X}\right)=\overline{\mathbf{K}}_{\mathfrak{P}}(\mathbf{Z}, \mathbf{X})
\end{aligned}
$$

$(\stackrel{(n)}{\approx}$ denoting the isomorphisms $n=(6)$ or $(7)$ of Corollary 2.3). In addition we have used the fact that there exists (in contrast to the case of cossmorphisms) by definition an isomorphism

$$
\overline{\mathbf{K}}_{\mathfrak{P}}(A, B \bar{\wedge} C) \approx \overline{\mathbf{K}}_{\mathfrak{P}}(A, C \bar{\wedge} B)
$$

whenever both sides are defined.

As a result we obtain

$$
\mathfrak{P}^{\prime}(D \mathbf{X}, D \mathbf{Z}) \approx \overline{\mathbf{K}}_{\mathfrak{P}}(\mathbf{Z}, \mathbf{X}),
$$

yielding another formulation of Theorem 1.1 of [1] (now for based spaces).

4) The first attempt to extend S-duality over the category of finite polyhedra was successfully made by E. Lima [7]. He anticipated stable ordinary shape theory (ten years before this notion was explicitly introduced). The associated contention for strong shape theory, which is an immediate consequence of Alexander duality, constitutes the main result of [2] (Theorem 
1.1; cf. [1, §1] for a correction). This theorem goes back to Q. Haxhibeqiri and S. Nowak [6], who proved it by using different methods.

On the other hand, there were negative results, asserting that there is principally no S-duality for categories like the Boardman category [4]. A second non-existence proof for S-duality emerges from a result by T. Lin [8]. His argument applies to any category which is stable and contains sufficiently many Eilenberg-MacLane objects.

Crucial for all these non-existence proofs is the validity of a Whitehead theorem: A map inducing isomorphisms of homotopy groups is a homotopy equivalence. In our category coss, as in all kinds of shape categories, we do not have such a Whitehead theorem.

3. The S-dual $D$ and some S-categories. Since, in contrast to [1], we are dealing with based spaces $X=\left(X, x_{0}\right)$, we define for $\left(X, x_{0}\right), X \subset S^{n}$,

$$
D_{n} X=\left(\left(S^{n} \backslash\left\{x_{0}\right\}\right) \cup C\left(S^{n} \backslash X\right), \star\right),
$$

where $C(\ldots)$ denotes the unreduced cone over ... and $\star$ the vertex of this cone. Employing the cone $\widetilde{C}$ with the ordinary topology we have analogously

$$
\widetilde{D}_{n} X=\left(S^{n} \backslash\left\{x_{0}\right\}\right) \cup \widetilde{C}\left(S^{n} \backslash X\right)
$$

observing that $\widetilde{D}_{n} X$ and $D_{n} X$ are naturally homotopy equivalent.

Since $S^{n} \backslash\left\{x_{0}\right\} \subset S^{n} \backslash\left\{x_{0}\right\} \cup \widetilde{C}\left(S^{n} \backslash X\right)$ is closed and, in addition, a cofibration, we have

Proposition 3.1. In Met there exists a homotopy equivalence

$$
D_{n} X \simeq\left(\Sigma\left(S^{n} \backslash X\right), \star\right)
$$

$(\star=$ top vertex of $\Sigma)$.

Proof. Follows for $\widetilde{D}_{n}$ and $\widetilde{\Sigma}$, thus for $D_{n}$ and $\Sigma$.

This allows us to retain for based $\left(X, x_{0}\right)$ Corollary 3.4 of [1]:

Proposition 3.2. If $X \subset S^{n} \subset S^{n+1}$ ( $S^{n}=$ equator of $\left.S^{n+1}\right), X=$ $\left(X, x_{0}\right)$, is an embedding, then in $\operatorname{coss}$ we have natural equivalences

$$
\begin{aligned}
\Sigma D_{n} X & \approx D_{n+1} X, \\
D_{n+1} \Sigma X & \approx D_{n} X, \\
D_{n+1} D_{n} X & \approx \Sigma X .
\end{aligned}
$$

COROLlary 3.3. If $X$ in 3.2 is compact, then the equivalences (1)-(3) hold already in $\mathbf{M e t}_{h}$ (i.e. they are ordinary homotopy equivalences).

Proof. The fact that (1) is an ordinary homotopy equivalence is verified in $[1,3.5]$. Concerning (2) we have (cf. $[1, \S 5$, and Corollary 3.5]) 


$$
D_{n+1}(X \times I) \approx D_{n+1} X \text { and } D_{n+1}(X \times I) \approx \Sigma D_{n} X .
$$

Finally, (3) follows from (2) immediately as in the proof of [1, 3.4].

$D_{n}$ turns out to be compatible with the $\wedge$-product. More precisely:

Proposition 3.4. For $\left(X, x_{0}\right),\left(Y, y_{0}\right) \in$ Met with $X \subset S^{n}$ and $Y \subset S^{m}$, we have a homotopy equivalence

$$
D_{n} X \wedge D_{m} Y \simeq D_{n+m}(X \wedge Y)
$$

$\left(X \wedge Y \subset S^{n} \wedge S^{m} \approx S^{n+m}\right)$.

Proof. We show the argument for $\widetilde{D}_{n}, \widetilde{D}_{m}$ and $\widetilde{\wedge}$ instead of $D_{n}, D_{m}$ and $\wedge$ (cf. $\S 1$ and $[1, \S 1])$; since both are homotopy equivalent, the assertion follows.

We compare

$$
\widetilde{D}_{n} X \widetilde{\wedge} \widetilde{D}_{m} Y=\left(S^{n} \backslash\left\{x_{0}\right\} \cup \widetilde{C}\left(S^{n} \backslash X\right)\right) \widetilde{\wedge}\left(S^{m} \backslash\left\{y_{0}\right\} \cup \widetilde{C}\left(S^{m} \backslash Y\right)\right)
$$

with

$$
\widetilde{D}_{n+m}(X \widetilde{\wedge} Y)=S^{n+m} \backslash\left\{x_{0}, y_{0}\right\} \cup \widetilde{C}\left(S^{n+m} \backslash X \widetilde{\wedge} Y\right)
$$

by specifying various kinds of points in both spaces.

Adopting the notation: $\widetilde{x} \in S^{n} \backslash\left\{x_{0}\right\}, \widetilde{y} \in S^{m} \backslash\left\{y_{0}\right\}, \bar{x} \in S^{n} \backslash X$, $\bar{y} \in S^{m} \backslash Y$, we obtain

$$
[\widetilde{x}, \widetilde{y}] \in S^{n} \backslash\left\{x_{0}\right\} \widetilde{\wedge} S^{m} \backslash\left\{y_{0}\right\} \quad \text { resp. in } S^{n+m} \backslash\left\{x_{0}, y_{0}\right\}
$$

(observing that in the latter space we also find $\left[x_{0}, \widetilde{y}\right]=\star=\left[\widetilde{x}, y_{0}\right]$ ),

$$
\begin{aligned}
& {[\widetilde{x}, \bar{y}, t] \in S^{n} \backslash\left\{x_{0}\right\} \widetilde{\wedge} \widetilde{C}\left(S^{m} \backslash Y\right) \quad \text { resp. in } \widetilde{C}\left(S^{n+m} \backslash X \widetilde{\wedge} Y\right),} \\
& {[\bar{x}, \widetilde{y}, s] \in \widetilde{C}\left(S^{n} \backslash X\right) \widetilde{\wedge} S^{m} \backslash\left\{y_{0}\right\} \quad \text { resp. in } \widetilde{C}\left(S^{m+n} \backslash X \widetilde{\wedge} Y\right),} \\
& {[\bar{x}, \bar{y}, s, t] \in \widetilde{C}\left(S^{n} \backslash X\right) \widetilde{\wedge} \widetilde{C}\left(S^{m} \backslash Y\right), \quad s, t \in I .}
\end{aligned}
$$

The points of the last kind are those of the double cone over $S^{n} \backslash X \widetilde{\wedge} S^{m} \backslash Y$, which is a strong deformation retract of $\widetilde{C}\left(S^{n} \backslash X \widetilde{\wedge} S^{m} \backslash Y\right)=\{[\bar{x}, \bar{y}, t]\}$.

Since

$$
\{[\widetilde{x}, \bar{y}, t]\} \cup\{[\widetilde{x}, \widetilde{y}, s]\} \cup \widetilde{C}\left(S^{n} \backslash X \widetilde{\wedge} S^{m} \backslash Y\right)=\widetilde{C}\left(S^{m+n} \backslash X \widetilde{\wedge} Y\right),
$$

we conclude that (6) is a deformation retract of (5), thereby completing the proof of 3.4.

In $[1, \S 4]$ we introduced the S-category $\mathfrak{P}$, with pairs $\mathbf{X}=(X, m), m \in \mathbb{Z}$, $X \in$ Met finite-dimensional, as objects, whose morphisms are defined by means of coss-morphisms in a well-known way. This also works for based spaces and morphisms, so that we are able to transfer all results of $[1, \S 4]$ to our case. In particular, we obtain $D_{k} \mathbf{X}$ for any $k \geq 0, D \mathbf{X}=D_{0} \mathbf{X}$ and (as a consequence of $3.2(3)) D^{2} \mathbf{X} \approx \mathbf{X}$. We agree to write simply $X$ for $(X, 0)$ 
and call $\mathbf{X}=(X, m)$ compact (resp. good) whenever $X$ is compact (resp. good).

We have to define mappings

$$
\boldsymbol{\alpha}: \mathbf{X} \wedge \mathbf{Y} \rightarrow \mathbf{Z}, \quad \boldsymbol{\beta}: \mathbf{X} \rightarrow \mathbf{Y} \pi \mathbf{Z},
$$

for $\mathbf{X}=(X, m), \mathbf{Y}=\left(Y, m^{\prime}\right), \mathbf{Z}=\left(Z, m^{\prime \prime}\right)$. This is accomplished in the same way as in $[1, \S 4]$ :

Suppose that for suitable $k, l$ we have coss-morphisms

$$
\alpha: \Sigma^{k} X \bar{\wedge} \rightarrow \Sigma^{l} Z
$$

with $k+m+m^{\prime}=l+m^{\prime \prime}$, and

$$
\beta: \Sigma^{k} X \rightarrow \Sigma^{l} Y \pi Z
$$

with $k+m=m^{\prime}+m^{\prime \prime}+l$. Because coss-morphisms can be desuspended (cf. 1.7), we are allowed to confine ourselves to morphisms

$$
\begin{array}{ll}
\alpha: \Sigma^{m^{\prime}+m^{\prime \prime}-m} X \bar{\wedge} \rightarrow Z, & m^{\prime}+m^{\prime \prime}-m \geq 0, \\
\alpha: X \wedge Y \rightarrow \Sigma^{m-m^{\prime}-m^{\prime \prime}} Z, & m^{\prime}+m^{\prime \prime}-m \leq 0,
\end{array}
$$

and

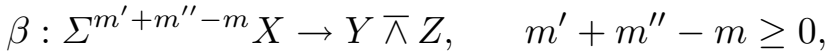

$$
\begin{aligned}
& \beta: X \rightarrow \Sigma^{m-m^{\prime}-m^{\prime \prime}} Y \wedge Z, \quad m^{\prime}+m^{\prime \prime}-m \leq 0 .
\end{aligned}
$$

So we define

and

$$
\{\mathbf{X}, \mathbf{Y} \wedge \mathbf{Z}\}_{c}= \begin{cases}\left\{\Sigma^{m^{\prime}+m^{\prime \prime}-m} X, Y \bar{\wedge}\right\}_{c} & \text { if } m^{\prime}+m^{\prime \prime}-m \geq 0 \\ \left\{X, \Sigma^{m-m^{\prime}-m^{\prime \prime}} Y \bar{\wedge}\right\}_{c} & \text { if } m^{\prime}+m^{\prime \prime}-m \leq 0\end{cases}
$$

$$
\{\mathbf{X} \pi Y, \mathbf{Z}\}_{c}= \begin{cases}\left\{\Sigma^{m^{\prime}+m^{\prime \prime}-m} X \pi Y, Z\right\}_{c} & \text { if } m^{\prime}+m^{\prime \prime}-m \geq 0, \\ \left\{X \wedge Y, \Sigma^{m-m^{\prime}-m^{\prime \prime}} Z\right\}_{c} & \text { if } m^{\prime}+m^{\prime \prime}-m \leq 0 .\end{cases}
$$

Since according to 1.8 all $\{\ldots, \ldots\}_{c}=\operatorname{coss}(\ldots, \ldots)$ carry a natural abelian group structure which is preserved under suspension, we conclude

Proposition 3.5. $\{\mathbf{X} \wedge \mathbf{Y}, \mathbf{Z}\}_{c}$ as well as $\{\mathbf{X}, \mathbf{Y} \backslash \mathbf{Z}\}_{c}$ are endowed with an abelian group structure, which is natural with respect to coss-morphisms in $\mathfrak{P}$.

In addition to $\mathfrak{P}$ we need 1 ) the S-category $\mathfrak{P}^{\prime}$ with the same objects as $\mathfrak{P}$ but stable homotopy classes of continuous mappings (rather than cossmorphisms as in the case of $\mathfrak{P}$ ) as morphisms; 2) the S-category $\overline{\mathbf{K}}_{\mathfrak{P}}$ with compact $\mathbf{X}$ as objects and stable homotopy classes of strong shape morphisms as morphisms; 3 ) morphism sets $\overline{\mathbf{K}}_{\mathfrak{P}}(\mathbf{X}, \mathbf{Y} \backslash \mathbf{Z}$ ) (not forming a category) which are established by the same procedure as $\{\mathbf{X}, \mathbf{Y} \backslash \mathbf{Z}\}_{c}$ above, but with strong shape morphisms $X \rightarrow Y \overline{ } Z, X$ compact or good, $Z$ compact and $Y$ good, or $Y$ and $Z$ exchanged $(\S 4)$. 
We have the based version of Lemma 4.1 of [1]:

LEMma 3.6. Up to isomorphism in $\mathfrak{P}, D_{n} \mathbf{X}$ is independent of the particular embedding $X \subset S^{n}$ and therefore of the dimension $n$ which is used to establish a "geometrical" $D_{n} X$.

Lemma 3.7. For compact $\mathbf{X}$ the independence of $D_{k} \mathbf{X}$ is up to isomorphism in $\mathfrak{P}^{\prime}$. Moreover, the isomorphism $D^{2} \mathbf{X} \approx \mathbf{X}$ occurs already in $\mathfrak{P}^{\prime}$.

Proof. Follows from 3.3 in the same way as 3.6 (i.e. $[1,4.1]$ ) from $[1$, 3.3].

We define in $\mathfrak{P}$

$$
\mathbf{X} \wedge \mathbf{Y}=(X, n) \wedge(Y, m)=(X \wedge Y, m+n)
$$

obtaining

Proposition 3.8. We have in $\mathfrak{P}$ an equivalence

$$
D(\mathbf{X} \wedge \mathbf{Y}) \approx D \mathbf{X} \wedge D \mathbf{Y} .
$$

For $\mathbf{X}, \mathbf{Y}$ compact, this equivalence occurs already in $\mathfrak{P}^{\prime}$.

Proof. Follows from 3.2-3.4:

$$
\begin{aligned}
D(\mathbf{X} \wedge \mathbf{Y}) & =\Sigma^{-m-n} D_{n+m}(\mathbf{X} \wedge \mathbf{Y}) \approx \Sigma^{-n-m}\left(D_{n} \mathbf{X} \wedge D_{m} \mathbf{Y}\right) \\
& \approx \Sigma^{-n} D_{n} \mathbf{X} \wedge \Sigma^{-m} D_{m} \mathbf{Y}=D \mathbf{X} \wedge D \mathbf{Y},
\end{aligned}
$$

where $m$ and $n$ are dimensions such that $D_{n} X$ and $D_{m} Y$ are geometrically defined.

4. Shape morphisms and Alexander duality. In addition to shape morphisms $\bar{f}: X \rightarrow Y$ between compacta we have to deal with strong shape morphisms $\bar{f}: X \rightarrow B \bar{\wedge} Y$ (or $\bar{f}: X \rightarrow Y \bar{\wedge} B$ ) where $X$ is either compact or an ANR, $B \in \mathbf{A N R}$ and $Y$ is compact. This kind of shape morphism has been defined in [3].

It is a 2-functor $\bar{f}: \mathbf{P}_{B \overline{ }} \rightarrow \mathbf{P}_{X}$, where $\mathbf{P}_{X}$ is a 2-category with continuous $g: X \rightarrow P \in \mathbf{A N R}$ as objects and suitably defined 1- and 2-morphisms, while $\mathbf{P}_{B \wedge Y}$ is a 2-category with continuous mappings $g^{\prime}: B \wedge Y \rightarrow Q \in$ ANR as objects, which factorize over a $1 \wedge g: B \wedge Y \rightarrow B \wedge P(B \in \mathbf{A N R}$ is kept fixed). For details we refer to [3], where in particular A5-A8, p. 298, ensure that we are allowed to confine ourselves to a subcategory $\mathbf{P}_{B \pi Y}^{\prime}$, with objects of the form $1 \wedge g_{m}: B \wedge Y \rightarrow B \wedge C_{m}, C_{m}$ being a compact ANR, $m=1,2, \ldots$

In the same way we define $\bar{f}: X \rightarrow Y \bar{\wedge} B$.

The set of all these strong shape morphisms (resp. all their stable homotopy classes) is denoted by $\overline{\mathbf{K}}(X, B \bar{\wedge} Y)$ and $\overline{\mathbf{K}}(X, Y \bar{\wedge} B)$ (resp. $\overline{\{X, B \bar{\wedge} Y\}}$ $=\overline{\mathbf{K}}_{s}(X, B \bar{\wedge} Y)$ and $\left.\overline{\{X, Y \bar{\wedge} B\}}=\overline{\mathbf{K}}_{s}(X, Y \bar{\wedge} B)\right)$. 
There are three different versions of Alexander duality which are used throughout this paper. We will assume of course that all spaces $E_{k}$ in a spectrum $\mathbf{E}=\left\{E_{k}\right\}$ are in Met.

Theorem 4.1. Let $\mathbf{E}=\left\{E_{k}\right\}$ be a spectrum and $\left(X, x_{0}\right)=X \subset S^{n} a$ based compact space. Then we have a natural (with respect to inclusions) isomorphism

$$
\overline{\mathbf{E}}_{p}\left(X, x_{0}\right) \approx \overline{\mathbf{E}}^{q}\left(S^{n} \backslash\left\{x_{0}\right\}, S^{n} \backslash X\right), \quad p+q=n, p, q \in \mathbb{Z} .
$$

TheOREM 4.2. Suppose $\mathbf{E}=\left\{E_{k}\right\}$ is a spectrum with compact $E_{k}$ and $\left(X, x_{0}\right)=X \subset S^{n}$ a based compact space. Then we have a natural (with respect to inclusions) isomorphism

$$
\overline{\mathbf{E}}_{p}\left(\Sigma\left(S^{n} \backslash X\right), \star\right) \approx \overline{\mathbf{E}}^{q}\left(X, x_{0}\right), \quad p+q=n, p, q \in \mathbb{Z} .
$$

In 4.1 and 4.2 , (co-) homology $\overline{\mathbf{E}}_{p}(X), \overline{\mathbf{E}}^{q}(Y)$ is always defined by using strong shape morphisms, e.g. $S^{n+k} \rightarrow X^{+} \pi E_{k}$ for homology and $\Sigma^{k} Y \rightarrow$ $E_{k+q}$ for cohomology, resp. in the based case (cf. [3, §3, §5], for details).

In order to formulate the next Alexander duality theorem, we take a compact spectrum $\mathbf{E}^{\prime}=\left\{E_{k}^{\prime}\right\}$ as in 4.2 and a good space $B \in$ Met. Now we introduce a formal spectrum $\mathbf{E}=\left\{B \bar{\wedge} E_{k}^{\prime}\right\}=B \bar{\wedge} \mathbf{E}^{\prime}$, with pairs of spaces $B \bar{\wedge} E_{k}^{\prime}$ and mappings of pairs of spaces

$$
\sigma_{k}=1 \pi \sigma_{k}^{\prime}: B \pi \Sigma E_{k}^{\prime} \rightarrow B \bar{\wedge} E_{k+1}^{\prime}
$$

as bonding maps. Alternatively we could realize all this by talking about virtual spaces $B \bar{\wedge} E_{k}$ and (componentwise defined) mappings between these virtual spaces (cf. remark at the end of $\S 1$ ).

This entitles us to form for compact $X \subset S^{n}$ (co-) homology $\overline{\mathbf{E}}_{p}\left(S^{n} \backslash X\right)$, $\overline{\mathbf{E}}^{q}(X)$ by using strong shape morphisms

$$
S^{p+k} \rightarrow\left(\left(S^{n} \backslash X\right)^{+} \wedge B\right) \pi E_{k}^{\prime} \quad \text { and } \quad \Sigma^{k} X \rightarrow B \bar{\wedge} E_{q+k}^{\prime} .
$$

The relative groups are defined in the same way. We have e.g.

$$
\overline{\mathbf{E}}_{p}\left(S^{n} \backslash X\right)=\overline{\mathbf{E}}_{p}^{\prime}\left(\left(S^{n} \backslash X\right) \wedge B\right) .
$$

We can of course also introduce formal spectra $\mathbf{E}=\mathbf{E}^{\prime} \pi B$.

TheOREM 4.3. Let $\mathbf{E}=B \pi \mathbf{E}^{\prime}$ be such a formal spectrum. Then for compact based $\left(X, x_{0}\right)=X \subset S^{n}$ we have the same isomorphism as in 4.2 :

$$
\overline{\mathbf{E}}_{p}\left(\Sigma\left(S^{n} \backslash X\right), \star\right) \approx \overline{\mathbf{E}}^{q}\left(X, x_{0}\right), \quad p+q=n, p, q \in \mathbb{Z} .
$$

All these duality isomorphisms are also natural with respect to mappings of spectra (in a well-known way; cf. 4.5 below).

Theorem 4.1, being a classical Alexander isomorphism, is identical with [3, Theorem 7.1]; Theorem 4.2(2) is identical with Theorem 1.2(2) of [2].

Only Theorem 4.3 is new. However, the proof is virtually identical with that of 4.2 in $[2, \S 3]$ : One simply has to replace the 2-category $\mathbf{P}_{Y}^{\prime}, Y=E_{q+k}$, 
by $\mathbf{P}_{B \wedge Y}^{\prime}$. Then all conclusions in $[2$, p. 20] carry through without any change.

We reformulate (1):

COROLlaRY 4.4. Under the same assumptions as in 4.1 we have for based compacta $\left(X, x_{0}\right)$ a natural isomorphism

$$
\overline{\mathbf{E}}_{p}\left(X, x_{0}\right) \approx \overline{\mathbf{E}}^{q}\left(\Sigma\left(S^{n} \backslash X\right), \star\right), \quad p+q=n, p, q \in \mathbb{Z},
$$

where, as usual, $\star=$ top vertex of the unreduced suspension.

Proof. There are isomorphisms

$$
\begin{aligned}
\overline{\mathbf{E}}^{q}\left(S^{n} \backslash\left\{x_{0}\right\}, S^{n} \backslash X\right) & \approx \overline{\mathbf{E}}^{q}\left(S^{n} \backslash\left\{x_{0}\right\} \cup C\left(S^{n} \backslash X\right), C\left(S^{n} \backslash X\right)\right) \\
& \approx \overline{\mathbf{E}}^{q}\left(S^{n} \backslash\left\{x_{0}\right\} \cup C\left(S^{n} \backslash X\right), \star\right) \\
& \approx \overline{\mathbf{E}}^{q}\left(\Sigma\left(S^{n} \backslash X\right), \star\right) ;
\end{aligned}
$$

the first isomorphism is an excision (one cuts off the top of the cone); the second stems from the exact cohomology sequence and the third from 3.1.

For suspension spectra $\mathbf{E}=\left\{\Sigma^{k} A\right\},\left\{\Sigma^{k}\right\},\left\{\Sigma^{k} Y \pi B\right\}$, we translate $\left(1^{\prime}\right)$, (2), (3) into a stable form:

$\left(1^{\prime \prime}\right) \quad \overline{\left\{S^{p}, X \bar{\wedge} A\right\}} \approx \overline{\left\{\Sigma^{p} D X, A\right\}}, \quad X$ compact, $A$ arbitrary,

$\left(2^{\prime}\right) \quad \overline{\left\{S^{-p}, D X \pi Y\right\}} \approx \overline{\left\{\Sigma^{-p} X, Y\right\}}, \quad X, Y$ compact,

$\left(3^{\prime}\right) \quad \overline{\left\{S^{-p},(D X \wedge B) \wedge Y\right\}} \approx \overline{\left\{S^{-p}, D X \wedge(Y \wedge B)\right\}}$

$$
\approx \overline{\left\{\Sigma^{-p} X, Y \bar{\wedge}\right\}}, \quad X, Y \text { compact, } B \text { good, }
$$

and in all three cases $p \in \mathbb{Z}$.

As a result we obtain, replacing $X$ by $\Sigma^{p} X$, in the same order and for the same kind of spaces:

$$
\begin{aligned}
& \overline{\left\{S^{0}, X \bar{\wedge} A\right\}} \approx \overline{\{D X, A\}}, \\
& \overline{\left\{S^{0}, D X \wedge Y\right\}} \approx \overline{\{X, Y\}}, \\
& \overline{\left\{S^{0},(D X \wedge B) \bar{\wedge}\right\}} \approx \overline{\left\{S^{0}, D X \bar{\wedge}(Y \bar{\wedge} B)\right\}} \approx \overline{\{X, Y \bar{\wedge} B\}} .
\end{aligned}
$$

In applications we encounter $A=P \wedge Y, P$ good, $Y$ compact.

Proposition 4.5. The isomorphisms (I)-(III) are natural with respect to stable homotopy classes of strong shape morphisms $\bar{f}: X \rightarrow X^{\prime}, \bar{g}: Y \rightarrow Y^{\prime}$ and continuous mappings $h: P \rightarrow P^{\prime}, h^{\prime}: B \rightarrow B^{\prime}$ for good $B, B^{\prime}, P, P^{\prime}$. Since $P^{\prime}, B^{\prime}$ are assumed to be good, the mappings $h, h^{\prime}$ can be replaced by strong shape mappings.

P r o o f. First of all, $\left(1^{\prime \prime}\right),\left(2^{\prime}\right),\left(3^{\prime}\right)$ are natural with respect to stable mappings between coefficient spectra (cf. also [3, §6, p. 267]) and with respect to inclusions for the spaces $X$. Now we proceed as in $[1, \S 1]$ and factorize a 
strong shape morphism $\bar{f}$ as in $[1, \S 1(3)]$. The same argument which established [1, Corollary 1.2] assures us that for given embeddings $X, X^{\prime} \subset S^{n}$ ( $n$ fixed) the isomorphisms $\left(1^{\prime \prime}\right),\left(2^{\prime}\right),\left(3^{\prime}\right)$ are natural with respect to strong shape morphisms.

The naturality properties of the stabilized versions (I)-(III) follow now from 3.6.

5. Some special properties of coss-morphisms. In $\S 1$ we introduced coss-morphisms $\beta: X \rightarrow Y \wedge Z$ by referring to strong shape morphisms of the form $X^{\prime} \rightarrow Q \bar{\wedge} Z^{\prime}$ and $P \rightarrow \widetilde{Y} \pi R$ ( $X^{\prime}, \widetilde{Y}, Z^{\prime}$ compact, $P, Q, R$ good). Therefore we have to investigate whether

1) for $X, Z$ compact and $Y$ good, and

2) for $X, Z$ good and $Y$ compact,

such a $\beta$ reduces to a strong shape morphism of this kind and vice versa.

Ad 1) Given a strong shape morphism $\bar{f}: X \rightarrow Y \bar{\wedge} Z$ (X,Z compact, $Y$ good) and $t: Z \rightarrow R \in \mathbf{A N R}$, we have a continuous $f: X \rightarrow Y \wedge R$ and an extension over some good $X \subset P$ (e.g. some open neighborhood in a Hilbert cube or some $S^{n}$ containing $X$ ) which, because $X$ is compact, can be assumed to be also compact: $f_{1}: P \rightarrow Y \wedge R$. Because $Z$ is compact, we can assume that $R$ is compact, so that $f_{1}$ maps already into some $\widetilde{Y} \wedge R$, with $\widetilde{Y} \subset Q=Y$ compact. This establishes $h: P \rightarrow \widetilde{Y} \wedge R$. Setting $X^{\prime}=X$, $Z^{\prime}=Z$ we obtain a $\bar{g}: X^{\prime} \rightarrow Q \bar{\wedge} Z^{\prime}(Q=Y)$, namely $\bar{g}=\bar{f}$. The same kind of argument provides us with an $\bar{h}: \widetilde{P} \rightarrow Y^{\prime} \wedge R$ (observing that we can assume $\left.X=X^{\prime}, P=\widetilde{P}\right)$.

Ad 2) Suppose that $X, Z$ are good and $Y$ compact and let $\bar{f}: X \rightarrow Y \bar{\wedge} Z$ be a strong shape morphism. Then we set $X=P=\widetilde{P}$ and $Y^{\prime}=\widetilde{Y}=Y$, providing us with $\widetilde{P} \rightarrow Y^{\prime} \bar{\wedge} R$ and $P \rightarrow \widetilde{Y} \bar{\wedge} R$. Moreover, let $s: Y \rightarrow Q \in$ ANR and $a: X^{\prime} \rightarrow X$ be given, with $X^{\prime}$ compact. Then composing with $\bar{f}$ yields (up to homotopy) a continuous $h_{1}: X^{\prime} \rightarrow Q \wedge Z=Q \wedge R$. By the same argument as in 1) we find a compact $Z^{\prime} \subset Z$ such that $h_{1}$ factorizes through $Q \wedge Z^{\prime}$, yielding a $\bar{g}: X^{\prime} \rightarrow Q \bar{\wedge} Z^{\prime}$.

In both cases it is clear that these morphisms establish a $\beta \in \operatorname{coss}(X$, $Y \wedge Z)$

On the other hand, let $\beta \in \operatorname{coss}(X, Y \pi Z)$ with either $X, Z$ compact, $Y$ good or $X, Z$ good and $Y$ compact. Then the existence of a strong shape morphism

$$
X=X^{\prime} \rightarrow Q \bar{\wedge} Z^{\prime}=Y \bar{\wedge} Z \text { and } X=P \rightarrow \widetilde{Y} \bar{\wedge} R=Y \bar{\wedge} Z
$$

is part of the definition.

Summarizing, we have: 
Proposition 5.1. For 1) $X, Z$ compact, $Y$ good, or 2) $X, Z$ good, $Y$ compact, there exists a natural isomorphism between $\operatorname{coss}(X, Y \bar{\wedge} Z)$ and the abelian group of all stable homotopy classes of strong shape morphisms

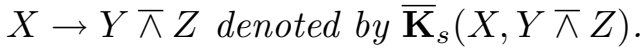

Concerning coss-morphisms $\alpha: X \bar{\wedge} Y \rightarrow Z$ we have a similar assertion:

Corollary 5.2. 1) For $X, Y, Z$ compact we have an isomorphism

$$
\operatorname{coss}(X \wedge Y, Z) \approx \overline{\mathbf{K}}_{s}(X \wedge Y, Z)=\overline{\{X \wedge Y, Z\}} .
$$

2) For $X, Y, Z$ good and $X$ or $Y$ compact we have an isomorphism

$$
\operatorname{coss}(X \wedge Y, Z) \approx \operatorname{Met}_{s}(X \wedge Y, Z) .
$$

Proof. Ad 1) Let $\alpha \in \operatorname{coss}(X \pi Y, Z)$ with $X, Y, Z$ compact. Then we can set $X=X^{\prime}, Y=Y^{\prime}$ and $Z=Z^{\prime}$, hence $X^{\prime} \wedge Y^{\prime} \rightarrow Z^{\prime}$ (as part of $\left.\alpha\left(X^{\prime}, Y^{\prime}, R\right)\right)$ gives a $\bar{g} \in \overline{\mathbf{K}}_{s}(X \wedge Y, Z)$.

Suppose on the other hand that $\bar{g} \in \overline{\mathbf{K}}_{s}(X \wedge Y, Z)$. Then by setting $X^{\prime}=X, Y^{\prime}=Y$ and $Z^{\prime}=Z$ we obtain a map $X^{\prime} \wedge Y^{\prime} \rightarrow Z^{\prime}$. Moreover, let $t: Z \rightarrow R \in \mathbf{A N R}$. Then

$$
X \wedge Y \rightarrow Z \rightarrow R
$$

can be assumed to be continuous, allowing an extension over some $P \wedge Q \in$ ANR, hence providing us with mappings $\widetilde{P} \wedge Q \rightarrow R$ and $P \wedge \widetilde{Q} \rightarrow R$ $(P=\widetilde{P}, Q=\widetilde{Q})$. According to Definition 1.1, these mappings determine an $\alpha \in \operatorname{coss}(X \pi Y, Z)$.

Ad 2) It suffices to treat the case of $X, Y, Z$ good and $Y$ compact, because of 1.3 .

Then according to $1.2(3)$,

$$
\operatorname{coss}(X \wedge Y, Z) \approx \operatorname{coss}(X \wedge Y, Z)
$$

Moreover, since $X, Y, Z$ are good, $[1,2.3]$ applies, yielding

$$
\operatorname{coss}(X \wedge Y, Z) \approx \operatorname{Met}_{s}(X \wedge Y, Z) .
$$

This completes the proof of 5.2 .

R e m a r k. There are of course stabilized versions of 5.1 and 5.2 operating with $\{\ldots, \ldots\}_{s}$ instead of $\operatorname{coss}(\ldots, \ldots), \overline{\mathbf{K}}_{\mathfrak{P}}$ instead of $\overline{\mathbf{K}}_{s}, \mathfrak{P}^{\prime}$ instead of $\mathbf{M e t}_{s}$ and $\mathbf{X}, \mathbf{Y}, \mathbf{Z}$ replacing $X, Y, Z$.

\section{References}

[1] F. W. Bauer, A strong shape theory admitting an S-dual, Topology Appl. 62 (1995), 207-232.

[2] - , A strong-shape theoretical version of a result due to E. Lima, ibid. 40 (1991), 17-21. 
[3] F. W. Bauer, Duality in manifolds, Ann. Mat. Pura Appl. (4) 136 (1984), 241-302.

[4] J. M. Boardman, Stable homotopy is not self-dual, Proc. Amer. Math. Soc. 26 (1970), 369-370.

[5] O. Hanner, Some theorems on absolute neighborhood retracts, Ark. Mat. 1 (1952), 389-408.

[6] Q. Haxhibeqiri and S. Nowak, Duality between stable strong shape morphisms and stable homotopy classes, Glas. Mat., to appear.

[7] E. Lima, The Spanier-Whitehead duality in new homotopy categories, Summa Brasil. Math. 4 (1959), 91-148.

[8] T. Y. Lin, Duality and Eilenberg-MacLane spectra, Proc. Amer. Math. Soc. 56 (1976), 291-299.

[9] H. R. Margolis, Spectra and the Steenrod Algebra, North-Holland Math. Library 29, North-Holland, 1983.

[10] E. Spanier, Algebraic Topology, McGraw-Hill, 1966.

Fachbereich Mathematik

Johann-Wolfgang-Goethe Universität

Robert-Mayer Str. 6-10

60054 Frankfurt a.M. 11, Germany

E-mail: f.w.bauer@mathematik.uni-frankfurt.d400.de

Received 25 January 1996 